\title{
Packet Error Probability in Multi-Path Diversity Scheme with Majority Combining
}

\author{
Vladimir Vuković, Grozdan Petrović, Member, IEEE, and Ljiljana Trajković, Fellow, IEEE
}

\begin{abstract}
In this paper, we derive the exact expression for the packet error probability in the case of $m$-path diversity scheme with packet combining using majority decision technique. The individual paths are modeled as independent and slow-varying binary symmetric channels with distinct values for bit error probabilities. We introduce an equivalent channel probability and spreading coefficients to analyze the derived expression. MATLAB code for numerical evaluations and an illustrative example for cases $m=3,5,7,9$, and 11 were also presented.
\end{abstract}

Index Terms-Majority combining, multi-path diversity scheme, packet error probability.

\section{INTRODUCTION}

$\mathbf{W}$ E consider a model of a multi-path diversity scheme with hard decision packet combining technique shown in Fig. 1. The transmitter and the receiver are connected with $m$ independent channels. Each channel is used for transmitting an identical copy of the frame containing the user packet $I_{T}$ and the corresponding header $H_{T}$ with additional bits for error detection, frame numeration, and identification. At the receiver side, the packet combining procedure is executed in two separate steps. In the first step, named selection combining (SC), received frame copies are individually processed. The transmission is declared successful and the corresponding packet $I_{R}$ is delivered to the user if at least one frame copy was received without detectable errors. If the SC procedure fails, the second step, named majority combining (MC), is activated. In that case, the combined frame generated by applying the bitby-bit majority decision criterion is checked. If the checksum is correct, the transmission is declared successful and the corresponding packet is delivered to the user. Otherwise, the packet is rejected.

Several authors [1]-[8] considered a similar model of the diversity transmission with majority combining technique in order to determine the packet error probability. In early works [1]-[5], the main motivation was to develop efficient hybrid automatic repeat request (ARQ) schemes. Recently, the evaluation of packet error probability has become interesting for determining the optimal energy balance in multipath and cooperative sensor networks [6]-[8]. Several authors

Manuscript submitted September 30, 2012; accepted October 24, 2012. The associate editor coordinating the review of this letter and approving it for publication was M. Matthaiou.

V. Vuković is with Department of Communication Networks, National Employment Service, Belgrade, Serbia (e-mail: vvukovic@nsz.gov.rs).

G. Petrović is with the Communications Department, Faculty of Electrical Engineering, University of Belgrade, Belgrade, Serbia (e-mail: gpetrovic@etf.bg.ac.rs).

Lj. Trajković is with the School of Engineering Science, Simon Fraser University, Vancouver, British Columbia, Canada (corresponding author, phone: (778) 782-3998; e-mail: ljilja@cs.sfu.ca).

Digital Object Identifier 10.1109/LCOMM.2013.13.122189 assumed that bit error probabilities in independent channels are equal [1]-[7]. The case with distinct channel probabilities and a general number of paths $m$ was also considered [8]. However, the evaluation of packet error probabilities was carried numerically based on a conceptually defined expression only.

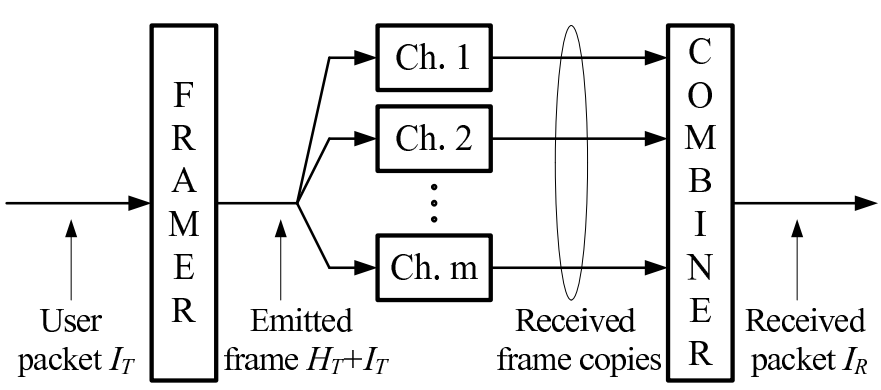

Fig. 1. Proposed model of the $m$-channel combining scheme.

In our prior work [9], we derived an exact analytical expression for the probability of packet error transmission for the case of distinct bit error probabilities and $m=3$. In this paper, our goal is to extend this result for the general case when $m$ is an odd integer $(m \geq 3)$. As in [9], we assume that: (a) channels are modeled as binary symmetric channels with the bit error probability $p_{i}=1-q_{i}(i=1, \ldots, m)$, (b) synchronization and alignment of the frame at the receiver are perfectly performed (i.e., recognizing the beginning and identifying each frame is perfect), and (c) the frame error detection procedure, usually based on the cyclic redundancy check, is perfect.

\section{Packet Error Probability}

Let $A_{i}(i=1, \ldots, m)$ denote the event when the transmission in a channel $i$ was performed without errors and let $B$ denote the event when the MC procedure was successful. The packet error probability may be expressed as [5]:

$$
P_{M}=1-\operatorname{Pr}\left\{A_{1} \cup A_{2} \cup \cdots \cup A_{m} \cup B\right\} .
$$

Let the complementary event be denoted by superscript $C$. By using identities $X \cup Y=Y \cup\left(X \cap Y^{C}\right)$ and $\operatorname{Pr}\left\{X^{C}\right\}=$ $1-\operatorname{Pr}\{X\}$, (1) may be rewritten as:

$$
P_{M}=\operatorname{Pr}\left\{B^{C}\right\}-\operatorname{Pr}\left\{\left(A_{1} \cup A_{2} \cup \cdots \cup A_{m}\right) \cap B^{C}\right\} .
$$


The principle of inclusion and exclusion [10] yields:

$$
\begin{aligned}
& P_{M}=\operatorname{Pr}\left\{B^{C}\right\}-\sum_{k=1}^{m}(-1)^{k} \\
& \cdot \sum_{1 \leq i_{1}<i_{2}<\cdots<i_{k} \leq m} \ldots \sum_{1} \operatorname{Pr}\left\{\left(A_{i_{1}} \cap A_{i_{2}} \cap \cdots \cap A_{i_{k}}\right) \cap B^{C}\right\}
\end{aligned}
$$

where $1 \leq i_{1}<i_{2}<\cdots<i_{k} \leq m$ denotes all possible combinations of class $k$ over the first $m$ natural numbers.

When all received frame copies are corrupted, which corresponds to the case $k=0$, probability $P_{M}=\operatorname{Pr}\left\{B^{C}\right\}$. Hence, (3) may be written as:

$$
\begin{gathered}
P_{M}=\sum_{k=0}^{m}(-1)^{k} \sum_{1 \leq i_{1}<i_{2}<\cdots<i_{k} \leq m} \operatorname{Pr}\left\{A_{i_{1}} \cap A_{i_{2}} \cap \cdots \cap A_{i_{k}}\right\} \\
\cdot \operatorname{Pr}\left\{B^{C} \mid\left(A_{i_{1}} \cap A_{i_{2}} \cap \cdots \cap A_{i_{k}}\right)\right\} .
\end{gathered}
$$

Taking into account that events $A_{i}$ are pair-wise independent and that $\operatorname{Pr}\left\{A_{i}\right\}=\left(1-p_{i}\right)^{L}$, it is easy to evaluate:

$$
\operatorname{Pr}\left\{A_{i_{1}} \cap A_{i_{2}} \cap \cdots \cap A_{i_{k}}\right\}=\left(q_{i_{1}} q_{i_{2}} \cdots q_{i_{k}}\right)^{L} .
$$

On the contrary, the evaluation of the conditional probability $\operatorname{Pr}\left\{B^{C} \mid\left(A_{i_{1}} \cap A_{i_{2}} \cap \cdots \cap A_{i_{k}}\right)\right\}$ is not trivial. It is based on Fig. 2, where $r[m]=\{1,2, \ldots, m\}$ denotes the set with $m$ received copies of the same frame. This set is divided into two subsets. The first subset $i_{m}[k]=\left\{i_{1}, i_{2}, \ldots, i_{k}\right\}$ consists of copies that are received without detectable errors while the second subset $i_{m}[k]^{C}=r[m] \backslash i_{m}[k]$ consists of copies that may contain errors. This second subset is decomposed into $L$ columns, where one column contains bits from distinct frames that belong to the same bit position. For example, Fig. 2 illustrates the $l$-th column with incorrectly transmitted bits that belong to the subset of received frame copies $j_{m-k}[s]=\left\{j_{1}, j_{2}, \ldots, j_{s}\right\} \subset i_{m}[k]^{C}$ while the remaining copies from the subset $i_{m}[k]^{C}$ have correctly transmitted bits in the $l$-th position.

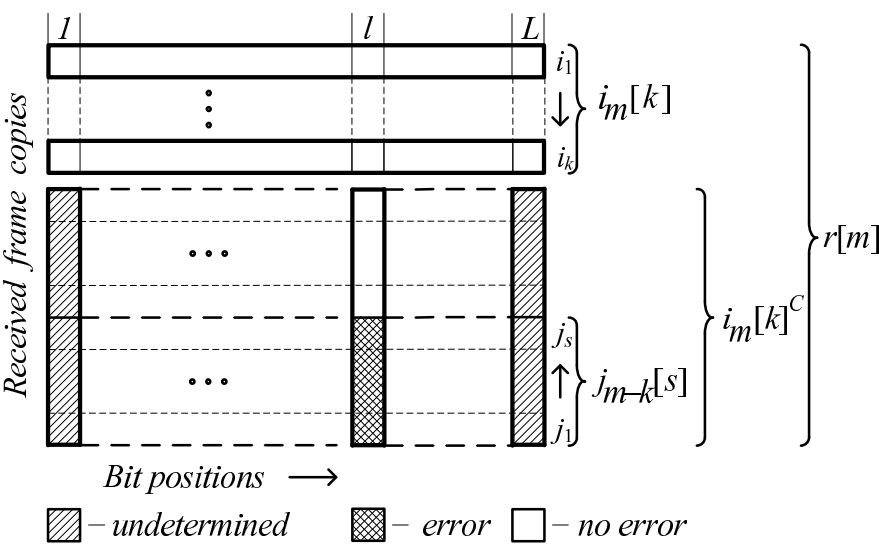

Fig. 2. Sample of stored copies and illustration of subsets $i_{m}[k]$ and $j_{m-k}[s]$.

The probability of an event that frame copies of subset $i_{m}[k]^{C}$ at an arbitrary bit position have less than $h+1$ errors is given by:

$$
P_{V}(h)=\sum_{s=0}^{h} \sum_{\substack{1 \leq j_{1}<\cdots<j_{s} \leq m \\ j_{1}, \ldots, j_{s} \neq i_{1}, \ldots, i_{k}}} \frac{q_{1} \cdots q_{m}}{q_{i_{1}} \cdots q_{i_{k}}} \cdot \frac{p_{j_{1}} \cdots p_{j_{s}}}{q_{j_{1}} \cdots q_{j_{s}}},
$$

where we assume that products $p_{j_{1}} \cdots p_{j_{s}}$ and $q_{j_{1}} \cdots q_{j_{s}}$ have value 1 when $s=0$.

The event $B^{C} \mid\left(A_{i_{1}} \cap A_{i_{2}} \cap \cdots \cap A_{i_{k}}\right)$ indicates the failure of the MC procedure after successful reception of copies from subset $i_{m}[k]^{C}$. Based on Fig. 2, we conclude that this event occurs when there is at least one bit position (i.e., column vector) with more than $m^{*}=(m-1) / 2$ errors. Since events corresponding to different bit positions are mutually independent, the probability of an event that copies from subset $i_{m}[k]^{C}$ have more than $m^{*}$ errors in at least one bit position is:

$$
\begin{aligned}
\operatorname{Pr}\left\{B^{C} \mid\left(A_{i_{1}} \cap A_{i_{2}} \cap \cdots \cap A_{i_{k}}\right)\right\} & \\
= & \begin{cases}0, & \text { if } k>m^{*} \\
1-P_{V}\left(m^{*}\right)^{L}, & \text { if } k \leq m^{*}\end{cases}
\end{aligned}
$$

Substituting (5) and (7) into (4), leads to:

$$
\begin{aligned}
& P_{M}=\sum_{k=0}^{m^{*}}(-1)^{k} \sum_{1 \leq i_{1}<\cdots<i_{k} \leq m}\left(q_{i_{1}} \cdots q_{i_{k}}\right)^{L} \\
& {\left[1-\left(\frac{q_{1} \cdots q_{m}}{q_{i_{1}} \cdots q_{i_{k}}} \cdot \sum_{s=0}^{m^{*}} \sum_{\substack{1 \leq j_{1}<\cdots<j_{s} \leq m \\
j_{1}, \ldots, j_{s} \neq i_{1}, \ldots, i_{k}}} \frac{p_{j_{1}} \cdots p_{j_{s}}}{q_{j_{1}} \cdots q_{j_{s}}}\right)^{L}\right] .}
\end{aligned}
$$

Expression (8) is the main result of this paper. For the case $m=3$, it leads to the previously reported result [9].

The procedure for numerical evaluation of the probability $P_{M}$ together with the related MATLAB code is given in the Appendix.

\section{Equivalent Channel Probability AND SPREADING COEFFICIENTS}

In this Section, we continue with the analysis of the packet error probability (8). After substituting $q_{k}=1-p_{k}$ and rearranging its right-hand side, we obtain the following polynomial form for $P_{M}$ :

$$
P_{M}=\sum_{j_{1}=1}^{L} \cdots \sum_{j_{m}=1}^{L} K_{\left\{j_{1}, \ldots, j_{m}\right\}} p_{1}^{j_{1}} \cdots p_{m}^{j_{m}}
$$

where $K_{\left\{j_{1}, \ldots, j_{m}\right\}}$ denote polynomial coefficients. The minimum value of the index $j_{i}$ is 1 and, as expected, $P_{M}=0$ if at least one frame copy is transmitted without errors. Hence, polynomial (9) may be rewritten in the form:

$$
P_{M}=K_{\{1, \ldots, 1\}} \cdot\left(p_{1} \cdots p_{m}\right) \cdot\left[1-F\left(p_{1}, \ldots, p_{m}\right)\right],
$$

where

$$
F\left(p_{1}, \ldots, p_{m}\right)=\sum_{\substack{1 \leq j_{1}, \ldots, j_{m} \leq L \\ j_{1} \cdots j_{m} \neq 1}} \frac{K_{\left\{j_{1}, \ldots, j_{m}\right\}}}{K_{\{1, \ldots, 1\}}} p_{1}^{j_{1}-1} \cdots p_{m}^{j_{m}-1} .
$$

Detailed analysis indicates that: (i) $F\left(p_{1}, \ldots, p_{m}\right)<1$ and (ii) $F\left(p_{1}, \ldots, p_{m}\right) \rightarrow 0$ when $p_{1}+\cdots+p_{m} \rightarrow 0$. Based 
on these properties, we conclude that product $p_{1} \cdots p_{m}$ has a dominant effect on the value of $P_{M}$, especially at low values of channel probabilities. This further suggests that the combined effect of $m$-channel transmission may be viewed using the "equivalent channel probability" $p_{E}$, which we define as:

$$
p_{E}=\left(p_{1} p_{2} \cdots p_{m}\right)^{1 / m} .
$$

Its maximum value is limited to 0.5 and, hence, individual channel bit error probabilities may be expressed as:

$$
p_{k}=\min \left\{x_{k} \cdot p, 0.5\right\},
$$

where $p(0 \leq p \leq 1)$ is the bit error probability and $x_{k}$ are "spreading coefficients" that satisfy constraints $x_{1} \cdots x_{m}=1$ and $x_{k}>0$.

\section{NUMERICAL EXAMPLE}

Equation (8) and results presented in Section III are used to calculate packet error probability $P_{M}$ as a function of equivalent channel probability $p_{E}$ and frame length $L$ for arbitrary number of paths $m(m=3,5,7,9$, and 11) and various distributions of spreading coefficients.

One numerical example is shown in Fig. 3. For illustration purposes, we choose the following distributions of spreading coefficients:

$$
x_{k}=D^{\frac{k-1}{m-1}-\frac{1}{2}}, \quad k=1, \ldots, m,
$$

with $D=1, D=10$, and $D=100$. In the case when $D$ $=1$, all spreading coefficients have identical values equal to 1. For other values of $D$ spreading coefficients are uniformly distributed on a logarithmic scale between $D^{-1 / 2}$ and $D^{+1 / 2}$.

Additional comprehensive analysis indicates that the probability $P_{M}$ increases with the increase of both the equivalent channel probability $p_{E}$ and the frame length $L$. Furthermore, $P_{M}$ significantly decreases when the number of channels $m$ increases. Considering the broader class of spreading coefficients for a fixed value of $p_{E}$, the dispersion of $P_{M}$ may become visibly large with increasing $m$ and $L$. However, this dispersion is relatively small when the product $p_{k} \cdot L$ is less than one.

\section{CONCLUding Remarks}

In this paper, we derived the exact analytical expression for the packet error probability $P_{M}$ in a diversity scheme that employs the majority packet combining for an arbitrary odd number of paths. For a given distribution of spreading coefficients, the existence of errors along individual paths may be expressed by the equivalent channel probability $p_{E}$, which is defined as a geometric mean of individual channel error probabilities.

We believe that the presented ideas and concepts may be successfully applied for calculating the packet error probability in cases when the number of paths is odd and for various majority voting decision criteria. Comparative analysis of possible other packet combining procedures is left for future work.
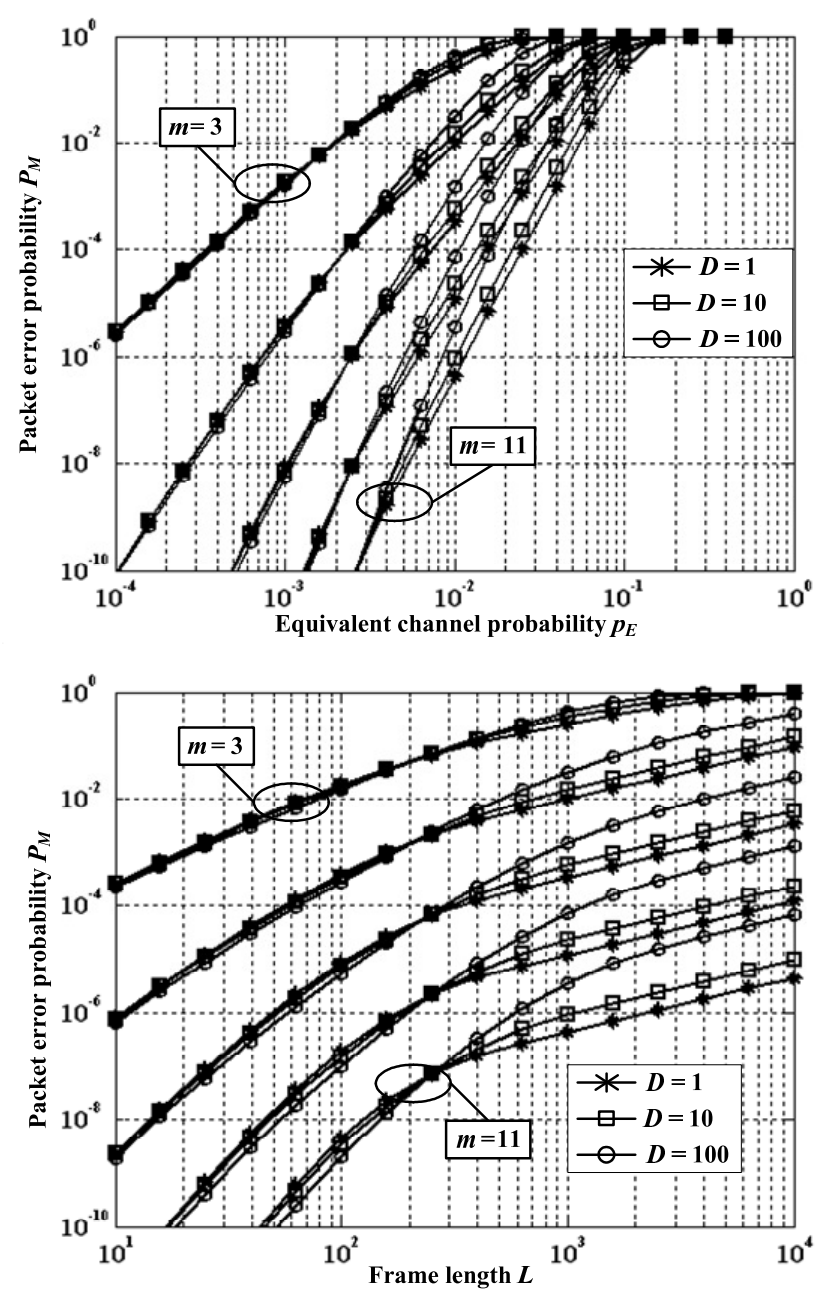

Fig. 3. Packet error probabilities as functions of: $p_{E}$ with $L=1,000$ (top) and $L$ with $p_{E}=0.01$ (bottom).

\section{APPENDIX}

Expression (8) may be replaced with the following sequence of equations:

$$
\begin{gathered}
P_{M}=\sum_{k=0}^{m^{*}}(-1)^{k} \cdot Y_{k} \\
Y_{k}=\sum_{i_{1}=1}^{m-k+1} \sum_{i_{2}=i_{1}+1}^{m-k+2} \ldots \sum_{i_{k}=i_{k-1}+1}^{m}\left[Q_{k}^{L}-H_{k}^{L}\right] \\
Q_{k}=\prod_{n=1}^{k} q_{i_{n}}, \quad k=1,2, \ldots, m^{*} \quad \text { and } \quad Q_{0}=1 \\
H_{k}=Q+\prod_{s=1}^{m^{*}} X_{s, k}, \quad k=0,1, \ldots, m^{*} \\
Q=\prod_{n=1}^{m} q_{n} \\
X_{s, k}=\sum_{\substack{1 \leq j_{1}<\ldots<j_{s} \leq m \\
j_{1}, \ldots, j_{s} \neq i_{1}, \ldots, i_{k}}} Q \cdot \prod_{n=1}^{s} \frac{p_{j_{n}}}{q_{j_{n}}}
\end{gathered}
$$


In order to avoid simultaneous multiplications and divisions by variable $q_{n}$ in individual sums (A.6), the following equivalent expression may be used:

$$
X_{s, k}=\sum_{j_{1}=1}^{m-s+1} \sum_{j_{2}=j_{1}+1}^{m-s+2} \cdots \sum_{j_{s}=j_{s-1}+1}^{m} \prod_{n=1}^{m} \tilde{q}_{n},
$$

where

$$
\tilde{q}_{n}=\left\{\begin{array}{ll}
q_{n}, & \text { if } n \notin\left\{j_{1}, \ldots, j_{s}\right\} \text { and } n \notin\left\{i_{1}, \ldots, i_{k}\right\} \\
p_{n}, & \text { if } n \in\left\{j_{1}, \ldots, j_{s}\right\} \text { and } n \notin\left\{i_{1}, \ldots, i_{k}\right\} \\
0, & \text { if } n \in\left\{i_{1}, \ldots, i_{k}\right\}
\end{array} .\right.
$$

(The subset $\left\{i_{1}, \ldots, i_{k}\right\}$ is empty if $k=0$.)

The calculation of $P_{M}$ is performed using three MATLAB subprograms named FunA1, FunA2, and FunA3 corresponding to (A.1), (A.2)-(A.5), and (A.7)-(A.8), respectively. Subprograms FunA2 and FunA3 are recursive and part of FunA1. Details explanations are omitted for the sake of brevity. Listings of these subprograms are shown.

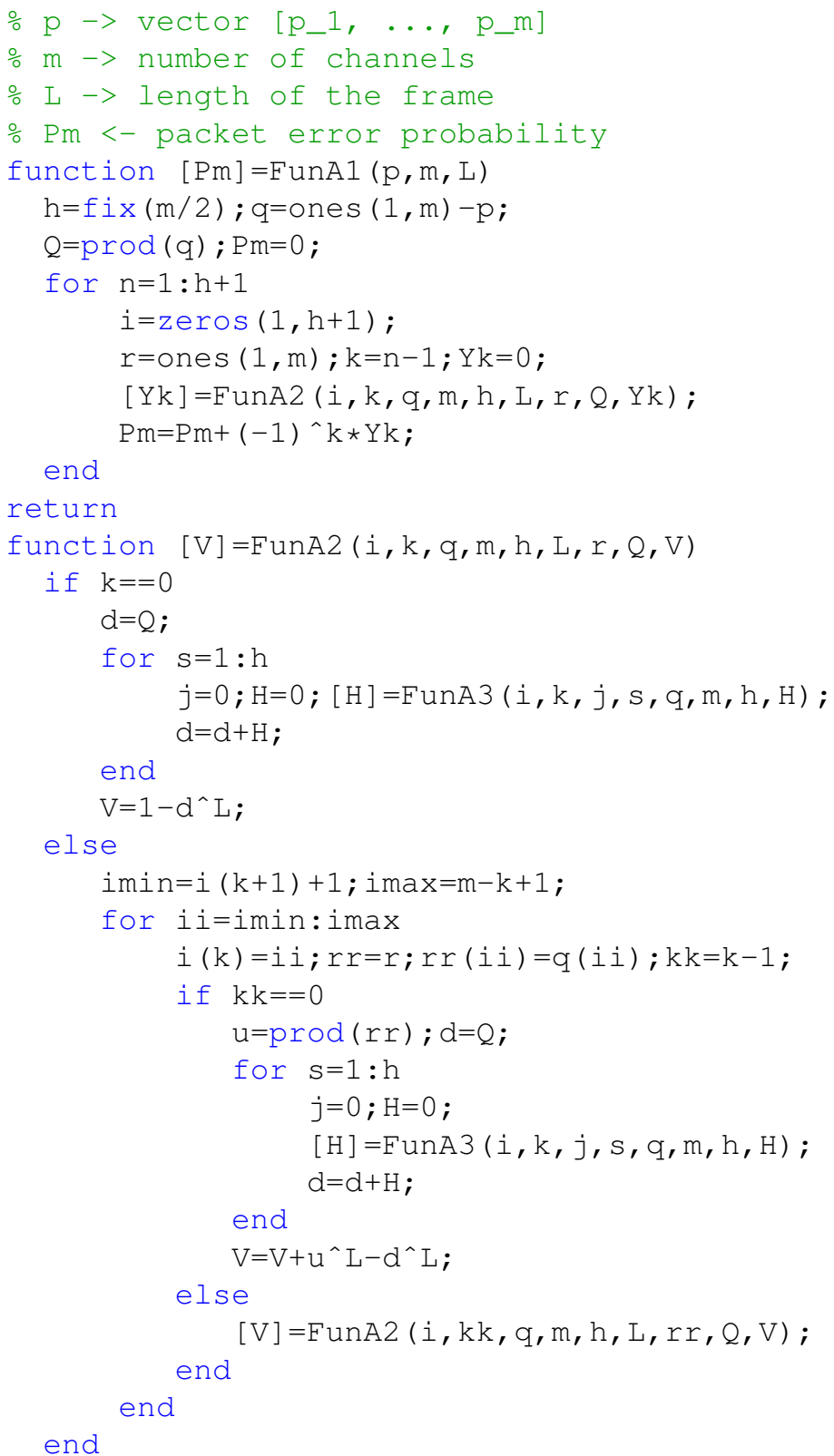

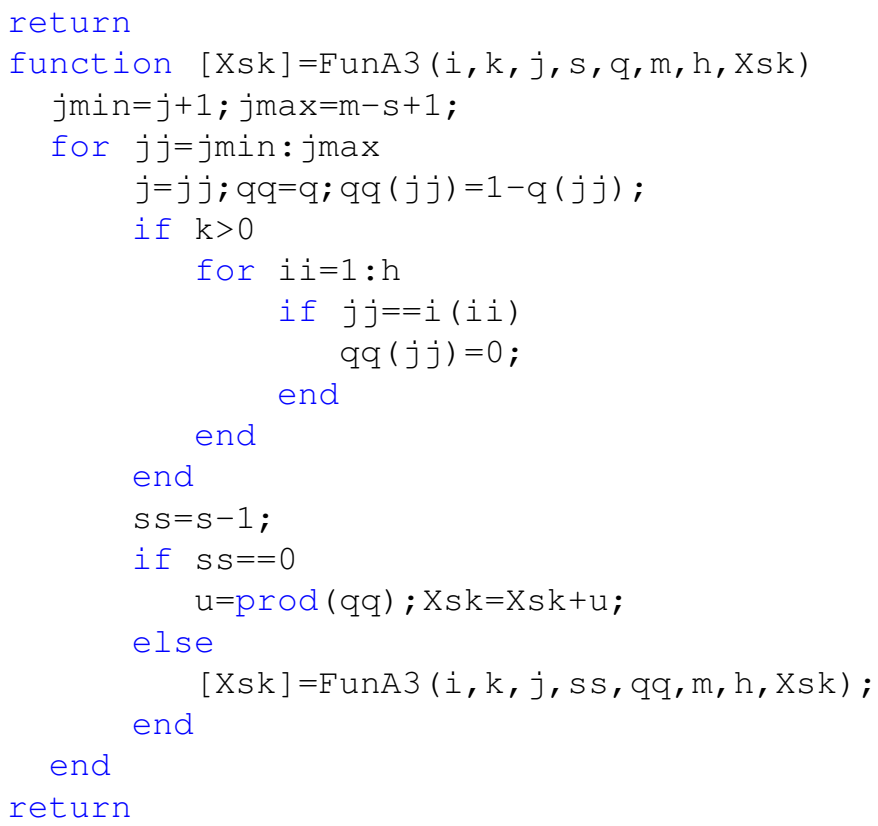

Numerical evaluations are performed using a desktop computer. The computation time does not depend significantly on values of channel bit error probabilities and frame lengths. However, it grows exponentially with the number of channels.

\section{ACKNOWLEDGMENT}

The authors would like to thank anonymous reviewers for their valuable comments and suggestions.

\section{REFERENCES}

[1] P. S. Sindhu, "Retransmission error control with memory," IEEE Trans. Commun., vol. COM-25, no. 5, pp. 473-479, May 1977.

[2] S. B. Wicker, "Adaptive rate error control through the use of diversity combining and majority-logic decoding in a hybrid-ARQ protocol," IEEE Trans. Commun., vol. 39, no. 3, pp. 380-385, Mar. 1991.

[3] Y. Liang and S. S. Chakraborty, "ARQ and packet combining with post-reception selection diversity," in Proc. IEEE Vehicular Technology Conference (VTC 2004-Fall), Los Angeles, CA, USA, Sept. 2004, vol. 3, pp. 1853-1857.

4] E. Masala, A. Servetti, and J. C. de Martin, "Standard compatible error correction for multimedia transmissions over 802.11 WLAN," in Proc. IEEE ICME 2005, Amsterdam, The Netherlands, July 2005, pp. 880883.

[5] R. Cam, C. Leung, and C. Lam, "Performance analysis of some harddecision combining schemes," IEEE Trans. Commun., vol. 42, no. 9, pp. 2650-2653, Sept. 1994.

[6] H. Okada, N. Koie, N. Nakagawa, T. Yamazato, and M. Katayama, "A study on error correcting and diversity combiner scheme on multiple routes in wireless multi-hop networks," in Proc. 1st Int. Symp. Wireless Commun. Syst., Mauritius, Sept. 2004, pp. 183-187.

[7] E. Uhlemann and A. Willig, "Hard decision packet combining methods for industrial relay networks," in Proc. Second Int. Conference on Commun. and Electron. (HUT-ICCE 2008), Hoi An, Vietnam, June 2008, 104-108.

[8] Z. Zhou, Z. Peng, J.-H. Cui, and Z. Shi, "Efficient multi-path communication for time-critical application in underwater acoustic sensor networks," IEEE/ACM Trans. Netw., vol. 19, no. 1, pp. 28-41, Feb. 2011.

[9] V. Vuković, G. Petrović, and Lj. Trajković, "Packet error probability in a three-branch diversity system with majority combining," IEEE Commun. Lett., vol. 15, no. 1, pp. 7-9, Jan. 2011.

[10] J. A. Anderson, Discrete Mathematics with Combinatorics, Second edition. Upper Saddle River, NJ, USA: Prentice Hall, 2003, pp. 464-466. 